\title{
Surgical treatment of solid hemangioblastomas of the posterior fossa: A report of 28 cases
}

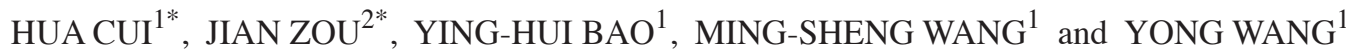 \\ ${ }^{1}$ Department of Neurosurgery, Renji Hospital, School of Medicine, Shanghai Jiaotong University, Shanghai 200127; \\ ${ }^{2}$ Department of Clinical Laboratory Science, Wuxi People's Hospital Affiliated to Nanjing Medical University, \\ Wuxi, Jiangsu 214023, P.R. China
}

Received April 7, 2015; Accepted October 7, 2016

DOI: $10.3892 / \mathrm{ol} .2016 .5531$

\begin{abstract}
This study evaluated the clinical features, treatment strategies and outcomes of solid hemangioblastomas in 28 patients diagnosed with hypervascular lesions in the posterior fossa. Preoperative embolization of the feeding arteries had limited effects, with only 7 patients benefitting from it for the reduction of intraoperative hemorrhage. The tumor was completely removed in all patients, and 22 patients had a full recovery, while 6 patients, all of whom had van Hippel Lindau disease, developed recurrences. The present study demonstrated that meticulous en bloc surgical resection was the optimal treatment for solid hemangioblastomas of the posterior fossa. For large tumors, preoperative embolization was critical for preventing postoperative morbidity. Given the improvements in microsurgical techniques and the understanding of the tumor vascular pattern, total tumor removal associated with a low mortality rate could be achieved.
\end{abstract}

\section{Introduction}

Hemangioblastomas of the central nervous system (CNS) are abundantly vascularized and benign neoplasms that are most commonly diagnosed in adults. They account for $1.5-2.5 \%$ of all intracranial tumors and for $7-12 \%$ of all posterior fossa tumors (1-3). Of all hemangioblastomas, $60-75 \%$ occur sporadically and $25-40 \%$ have a von Hippel-Lindau disease (VHL) background (4). Since, generally, hemangioblastomas do not metastasize, the World Health Organization categorizes them as grade one CNS tumors (4). Hemangioblastomas are

Correspondence to: Dr Yong Wang, Department of Neurosurgery, Renji Hospital, School of Medicine, Shanghai Jiaotong University, 1630 Dongfang Road, Shanghai 200127, P.R. China

E-mail: wenzhang1406@163.com

*Contributed equally

Key words: solid hemangioblastoma, posterior fossa, embolization, surgery either round or oval and have sharp boundaries and smooth contours. The majority of hemangioblastomas (70-75\%) have cystic or multi-cystic forms (4). These forms occur predominantly in the cerebellar hemispheres, while the solid forms of hemangioblastoma are found more frequently in the brain stem, cerebellar vermis and spinal cord (4). For the cystic type, the cavity has to be opened to expose the tumor nodule, which can be then removed by en bloc resection (3). Solid tumors have characteristics similar to intracranial arteriovenous malformations (AVMs) $(5,6)$.

Internal decompression and piecemeal resection have been shown to have potential devastating intraoperative complications (3). Therefore, the safe resection of solid tumors involves a sequence of surgical techniques, including preoperative embolization, wide exposure and circumferential dissection, which represent a challenge for the majority of neurosurgeons (3). Early case reports suggested that the postoperative mortality and morbidity rates for posterior fossa tumors were $\sim 50 \%$, while the intraoperative mortality rate was $9 \%(2,3,5)$. Generally, the high mortality and morbidity rates were closely associated with unsuccessful intraoperative hemostasis (7). Since the introduction of diagnostic imaging and modern treatment methods, the mortality and morbidity rates of hemangioblastomas have decreased significantly (8). The main risk factor for intraoperative hemorrhage is the tumor size, such that the surgical treatment of large solid hemangioblastomas remains relatively difficult (8). In our previous study, surgical resection was proposed as the optimal treatment for large tumors of the posterior fossa (8).

This study presents the results of microsurgeries performed between 2010 and 2014 on 28 patients with posterior fossa hemangioblastomas, and demonstrated that, with improved techniques and a better understanding of the vascular pattern of these tumors, total microsurgical removal can be performed with a relatively low mortality. Selective preoperative endovascular embolization of the feeding artery with subsequent en bloc tumor resection was shown to be the preferred procedure to reduce the risk of intraoperative hemorrhage and facilitate the removal of large solid tumors. However, embolization remains controversial, given its associated risks, including ischemia, bleeding and increased intracranial pressure (3). 


\section{Patients and methods}

Patients. This retrospective study included 28 consecutive patients with confirmed posterior fossa hemangioblastomas, treated between January 2010 and September 2014 at the Renji Hospital (Shanghai, China). Only patients with surgical treatment were included in this study. The therapeutic approach in all cases was surgical resection with or without preoperative endovascular embolization. Of the patients, 17 were males and 11 were females, with an age range of 15-85 years and a mean age of 40 years. In total, 21 tumors were sporadic and 7 were associated with VHL disease. The course of the disease ranged from 1 month to 4 years, with a mean disease duration of 1 year. All patients had varying degrees of intracranial hypertension symptoms (e.g. a headache); 9 patients had ataxia; 3 had diplopia; 2 had lower cranial nerve dysfunction; and 11 had preoperative hydrocephalus. The individual patient data are detailed in Table I.

Imaging and embolization. Brain computed tomography (CT) scans, magnetic resonance imaging (MRI) scans and digital subtraction angiography (DSA) were performed routinely for all patients. Tumors were divided into four types, based on their location: i) Type I was found in the cerebellar hemispheres and vermis; ii) type II was found in the cerebellar tonsil and lateral medulla; iii) type III was found in the fourth ventricle and brain stem; and iv) type IV was found in the superior vermis and dorsal midbrain. If the arteriography performed immediately following injection of the contrast solution (iopamidol) clearly indicated that the tumor had an abundant blood supply, selective embolization of the feeding arteries was performed preoperatively. The indication for preoperative embolization was decided on an individual basis, depending on the coagulation time of the feeding arteries when handling the nodule or the location of the nodule. All embolization procedures were performed by neuroendovascular specialists. The authors of the present study recommend that endovascular embolization be performed immediately or within 1 day of tumor removal, in order to avoid aggravation of edema in the tumor and the surrounding cerebellar tissue. However, if visualization by DSA was delayed, embolization of the feeding arteries was avoided. The feeding arteries could be divided into four types corresponding to the tumor classification: i) Type I was fed by the superior cerebellar artery (SCA), posterior inferior cerebellar artery (PICA) and anterior inferior cerebellar artery (AICA); ii) type II was fed by the AICA, PICA and meningeal branches; iii) type III was fed by the PICA; and iv) type IV was fed by the SCA and meningeal branches.

A general examination was conducted for the 7 patients with VHL disease and revealed three instances of renal cysts and one of retinal hemangioma.

Surgical procedure. The blood supply of hemangioblastomas is mainly derived from meningeal branches and intracranial feeding arteries; tumors in the cerebellar tonsil, lateral medulla, superior vermis and dorsal midbrain are fed by meningeal branches (4). During the early stages of surgery, the feeding arteries can be divided based on the various surgical approaches: The suboccipital ipsilateral approach, modified far-lateral approach and suboccipital midline approach were applied to tumor types I, II and III, respectively. Tumors, identified by microscopy intraoperatively, appeared as superficial racemose hemangiomas with a bright or dark red coloration. Intraoperative microvascular Doppler ultrasonography distinguished feeding arteries from draining veins. The authors of the present study believe that an early division of the draining veins may induce local swelling and influence the surgical procedure, and, therefore, they should be approached after the majority of the tumor has been dissected from the peripheral tissue.

The surgical principle for hemangioblastoma is similar to that for AVM (6). Total removal of the tumor is vital, and, thus, cleavage of the main draining veins, which are extremely dilated, should be performed at the very last moment. Surgery was performed under the principle that feeding arteries should be distinguished first and draining veins should be ligated last. The blood supply to the tumor was interrupted prior to resection, and complete resection was performed after the peripheral tumor tissue had been dissected (avoiding resection within the tumor). Finally, the draining vein was ligated. As internal decompression may cause uncontrollable bleeding (6), en bloc resection was carried out even for relatively large tumors. At the end of the operation, any bleeding was carefully handled by careful coagulation.

Illustrative case. A 36-year-old man (case 27) presented at the Renji Hospital with headaches, cerebellar ataxia, nystagmus and hearing difficulty in his right ear. The patient had a 1-month history of nausea and vertigo prior to operative exploration via the lateral suboccipital approach at the local hospital. A hypervascular tumor was identified intraoperatively, and, given its abundant blood supply, the therapeutic approach was a ventriculoperitoneal shunt, with recommendation for further treatment.

CT and MRI scans at our hospital revealed an intense mass in the right cerebellopontine angle (CPA), measuring $\sim 40 \mathrm{~mm}$ in diameter (Fig. 1A-C). The cerebral angiogram showed a hypervascular tumor fed by the right AICA and PICA (Fig. 2A). The patient underwent preoperative embolization of the feeding artery following the routine diagnostic angiography. All endovascular procedures were performed under general anesthesia. A microcatheter was inserted into the tumor feeders and the glue was delivered under image acquisition. The angiogram following embolization revealed a $90 \%$ reduction in the tumor vascularization (Fig. 2B). The day after embolization, complete surgical tumor resection was performed via a modified far-lateral approach (6). The embolized feeding arteries were visible as solid white vessels directly in the microscopic fields, which was useful for intraoperative orientation. The microcatheter failed to be withdrawn following embolization and was removed intraoperatively (Fig. 3). MRI showed no residual tumor at 3 weeks postoperatively (Fig. 4). The postoperative neurological examination did not reveal any neurological complication, such as facial palsy or auditory function and lower cranial nerve symptoms. Histological examination revealed typical hemangioblastomas consisting of numerous vessels, capillary mesh and stromal cells (Fig. 5). Tumor cells were fixed by formaldehyde and embedded in paraffin. Slice diameter was $5 \mu \mathrm{m}$. Histological analysis of the tumor specimen stained with hematoxylin and eosin revealed 
Table I. Clinical data of 28 patients with solid hemangioblastomas of the posterior fossa.

\begin{tabular}{|c|c|c|c|c|c|c|c|c|}
\hline No. & $\begin{array}{l}\text { Age }(y) / \\
\text { sex }\end{array}$ & Location $^{\mathrm{a}}$ & $\begin{array}{l}\text { Tumor } \\
\text { size } \\
(\mathrm{cm})\end{array}$ & $\begin{array}{c}\text { Initial } \\
\text { symptoms }\end{array}$ & $\begin{array}{l}\text { Tumor feeding } \\
\text { artery }\end{array}$ & $\begin{array}{l}\text { Preoperative } \\
\text { embolization }\end{array}$ & VHL & $\begin{array}{r}\text { Preoperative } \\
\text { hydrocephalus }\end{array}$ \\
\hline 1 & 29/M & I & 2.0 & Headache & SCA, PICA & - & + & - \\
\hline 2 & $44 / \mathrm{M}$ & I & 2.0 & Ataxia, nausea & PICA & - & - & - \\
\hline 3 & $44 / \mathrm{M}$ & I & 2.5 & Vertigo & AICA, SCA & - & + & + \\
\hline 4 & $85 / \mathrm{M}$ & III & 3.5 & Headache, diplopia & SCA, PICA & + & - & - \\
\hline 5 & 26/M & II & 2.5 & Ataxia & PICA & - & + & + \\
\hline 6 & 66/M & I & 3.5 & Headache & PICA, AICA, MB & - & + & + \\
\hline 7 & $37 / \mathrm{F}$ & I & 2.0 & Headache, ataxia & PICA AICA & - & - & - \\
\hline 8 & $43 / \mathrm{F}$ & I & 1.0 & Headache & AICA, SCA & - & - & - \\
\hline 9 & $28 / \mathrm{M}$ & III & 2.0 & Headache, ataxia & PICA AICA & - & - & - \\
\hline 10 & $41 / \mathrm{F}$ & III & 2.5 & Headache & SCA, PICA, MB & + & - & + \\
\hline 11 & $43 / \mathrm{F}$ & $\mathrm{I}$ & 2.0 & Headache, diplopia & AICA, SCA & - & - & - \\
\hline 12 & $27 / F$ & I & 1.5 & Headache & PICA, MB & - & - & - \\
\hline 13 & 49/M & I & 1.0 & Vertigo & PICA & - & + & - \\
\hline 14 & $53 / \mathrm{M}$ & I & 2.0 & Headache & PICA & - & - & + \\
\hline 15 & $36 / F$ & III & 2.5 & Ataxia, nausea & AICA, SCA & - & - & + \\
\hline 16 & $56 / \mathrm{M}$ & I & 3.0 & Headache & SCA, PICA & + & - & - \\
\hline 17 & 49/M & I & 2.0 & Headache & PICA AICA & - & - & - \\
\hline 18 & $33 / \mathrm{F}$ & I & 3.5 & Lower CN palsy & SCA, PICA & + & - & - \\
\hline 19 & 26/M & III & 3.0 & Ataxia & PICA, SCA & - & - & + \\
\hline 20 & $21 / \mathrm{F}$ & $\mathrm{I}$ & 3.0 & Ataxia, nystagmus & PICA & - & - & - \\
\hline 21 & $34 / F$ & $\mathrm{I}$ & 3.5 & Lower CN palsy & PICA AICA & + & + & + \\
\hline 22 & $15 / F$ & $\mathrm{I}$ & 1.0 & Headache, ataxia & PICA & - & - & + \\
\hline 23 & $22 / \mathrm{M}$ & I & 2.5 & Headache & AICA, SCA & - & - & - \\
\hline 24 & $42 / \mathrm{M}$ & II & 2.0 & Headache & PICA & - & - & - \\
\hline 25 & $25 / F$ & I & 1.5 & Nystagmus & PICA, MB & - & - & - \\
\hline 26 & $59 / \mathrm{M}$ & I & 2.0 & Ataxia, nystagmus & PICA & - & - & - \\
\hline 27 & 36/M & II & 4.0 & Ataxia, nystagmus & PICA AICA & + & - & + \\
\hline 28 & $60 / \mathrm{M}$ & I & 3.5 & Ataxia, diplopia & PICA & + & + & + \\
\hline
\end{tabular}

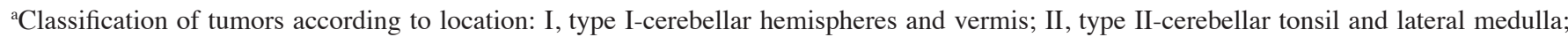
III, type III-fourth ventricle and brain stem; and IV, type IV-superior vermis and dorsal midbrain. AICA, anterior inferior cerebellar artery; F, female; M, male; No., number; PICA, posterior inferior cerebellar artery; SCA, superior cerebellar artery; MB, meningeal branch; VHL, von Hippel-Lindau disease; y, years; $\mathrm{CN}$, cranial nerve.
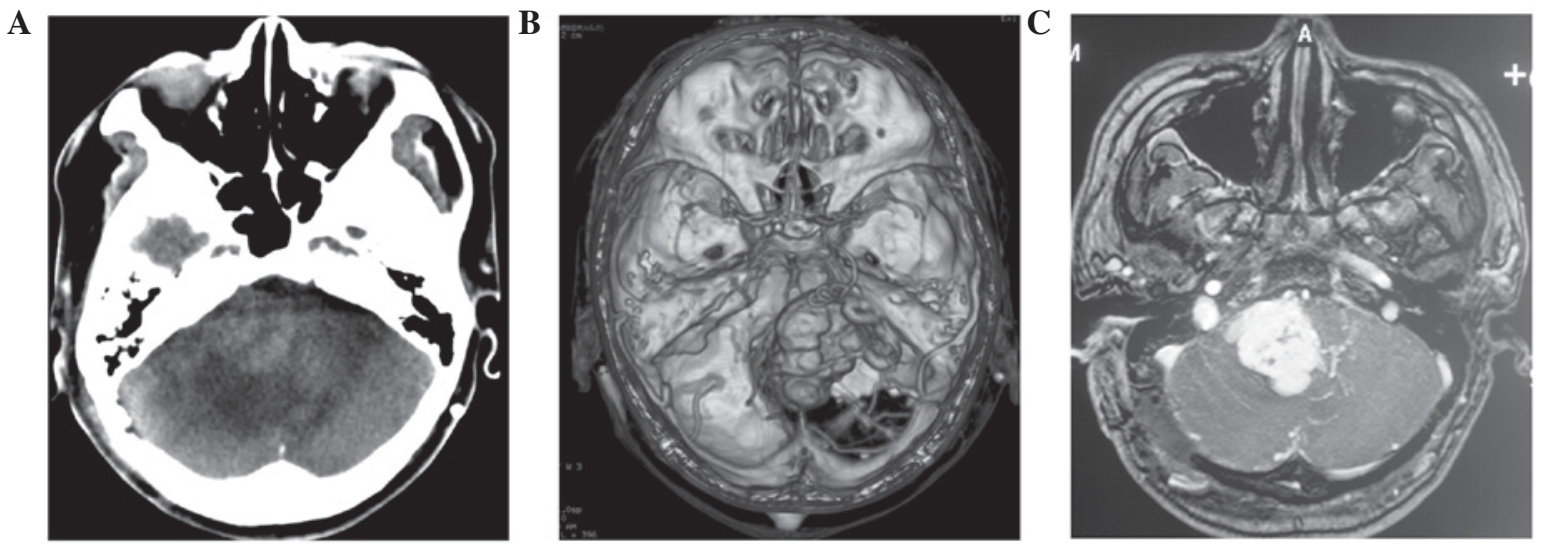

Figure 1. Preoperative CT and MRI scans of case 27. (A) CT scans revealed an isodense mass in the right CPA. (B) CT angiography showed a large hypervascular mass supplied by the right anterior inferior cerebellar artery and the posterior inferior cerebellar artery. (C) Preoperative T1-weighted MRI scan with gadolinium showing a heterogeneously enhanced lesion in the right CPA with multiple flow voids. CT, computed tomography; MRI, magnetic resonance imaging; CPA, cerebellopontine angle. 
A

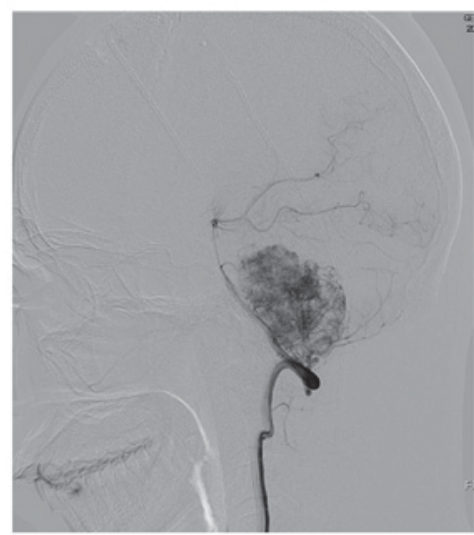

B

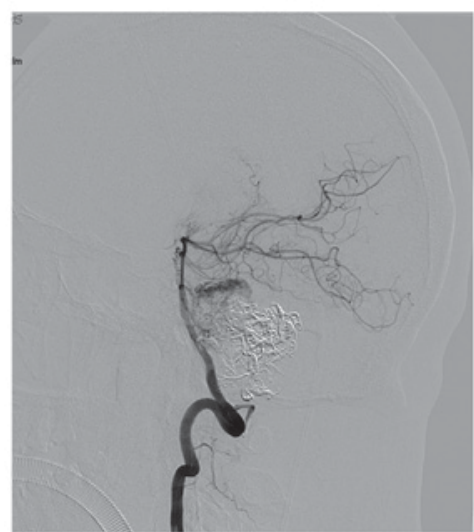

Figure 2. Digital subtraction angiography prior to and following embolization. (A) Right vertebral artery angiogram exhibits a hypervascular tumor fed by the anterior inferior cerebellar artery and the posterior inferior cerebellar artery before embolization. (B) The angiogram following the embolization shows a $90 \%$ reduction in tumor vascularization.

numerous vessels, a capillary mesh and vacuolated stromal cells, with large nuclei and an eosinophilic foamy cytoplasm with a light microscope. At a 6 month follow-up appointment, this patient's symptoms were markedly improved.

\section{Results}

Imaging results. The $\mathrm{CT}$ scans of the solid hemangioblastomas showed lesions that were either isodense or hyperdense, with clear margins. Enlarged feeding and draining vessels were visualized following contrast enhancement. The MRI scans showed low or isointense signals on T1-weighted MRI scans and relatively high signal intensities on T2-weighted MRI scans. In the majority of cases, abundant tumor feeding arteries that formed an intense vascular network around the mass were observed.

Patient outcomes. Preoperative embolization was achieved in 7 cases. The blood supply of the tumor was reduced significantly after embolization of the feeding arteries, without any related neurological deficits. In one case, the microcatheter could not be withdrawn following embolization and was removed during surgery. The tumor resection surgery was performed the day following embolization for all patients, in order to use embolized vessels as markers for intraoperative orientation.

En bloc resection was performed for all 28 patients. Postoperative pathological examinations of the resected tumors

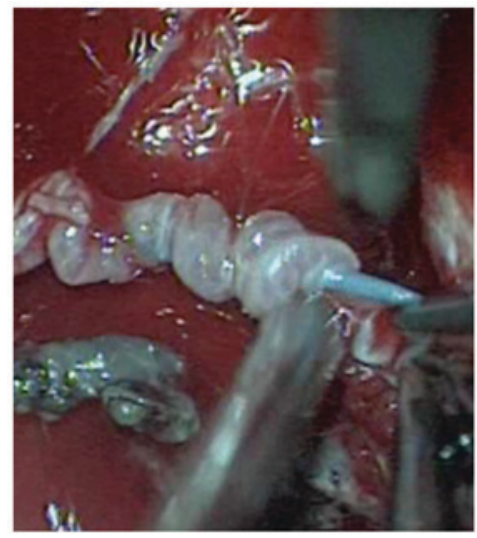

Figure 3. Microcatheter failed to be withdrawn following embolization in case 27 and was removed intraoperatively.

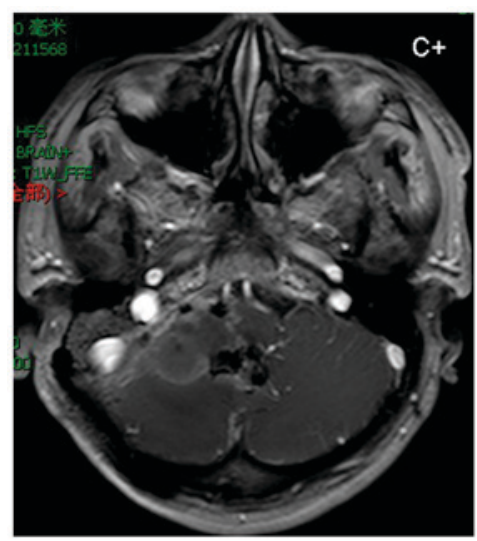

Figure 4. Postoperative gadolinium-enhanced T1-weighted magnetic resonance imaging confirmed complete resection of the hemangioblastoma.

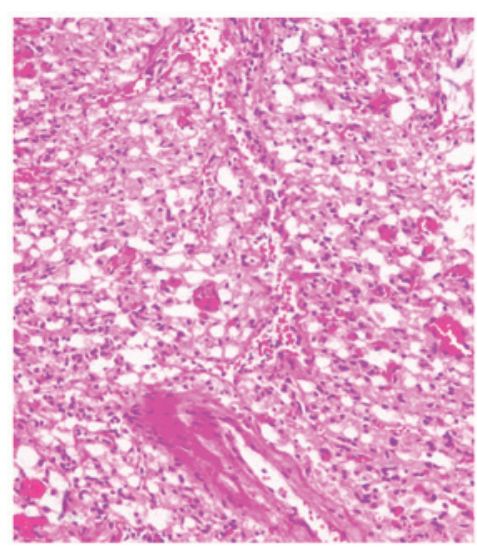

Figure 5. Histological analysis of the tumor specimen from case 27 stained with hematoxylin and eosin revealed numerous vessels, a capillary mesh and vacuolated stromal cells, with large nuclei and an eosinophilic foamy cytoplasm (magnification, $\mathrm{x} 100$ ).

confirmed that all were hemangioblastomas. Following surgery, 22 patients $(78.6 \%)$ showed an improvement of the neurological deficit. Of the remaining 6 patients (21.4\%), 2 showed neurological improvement or full recovery at the 3-month follow-up. Of the 7 patients with VHL disease, 6 presented with intracranial recurrences at other sites. There was no operative mortality. 


\section{Discussion}

Hemangioblastomas are pathologically benign neoplasms that originate predominantly from CNS tissues and should be completely removed. Clinically, about two-thirds appear as well-circumscribed cystic masses with hypervascular mural nodules (9). Although there are no histological differences between the cystic and solid tumor subtypes (2), solid hemangioblastomas have an increased risk of excessive intraoperative bleeding and postoperative complications (10). A careful strategy and a tailored approach should be considered for these cases. Based on our previous experiences, large solid subtypes are associated with a high surgical difficulty, given their AVM-like characteristics (7,11). Since most have the risk of uncontrollable intraoperative bleeding, they are hard to control, and thus, a preoperative intervention may be particularly important. Therefore, the present study aimed to reduce vascularization and achieve adequate operation space.

There are many ways to reduce the vascularization of a tumor. Kamitani et al (5) reported that radiosurgery at 9 months prior to a craniotomy significantly reduced the vascularization and subsequently enabled complete and safe tumor removal (5). Radiosurgery is also the elected approach for patients with small tumors, for patients with underlying medical conditions incompatible with surgery and for patients with multiple tumors (2). However, in a prospective study, Wan et al identified 2 patients with large hemangioblastomas who received $\gamma$-knife treatment, followed by surgery (8). They found that these tumors remained highly vascularized at 6-12 months following $\gamma$-knife treatment. In addition, they reviewed a number of cases that received radiotherapy as an adjuvant postoperative treatment, and found that the long-term outcome of tumor control was mostly poor. Since hemangioblastomas are generally considered to be radio-resistant tumors, this highlights the importance of preoperative embolization. In 7 of our cases, preoperative embolization was performed after a preoperative evaluation of the blood supply by DSA. The present study found that, following embolization, the tumors became firm, with clear boundaries, and were easy to orient. As a result, intraoperative bleeding was significantly decreased, the surgery time was shortened and the likelihood of total tumor resection was increased, which is consistent with previous studies $(3,8)$. However, preoperative embolization is associated with a high risk of cerebellar infarction and/or intratumoral hemorrhage, and makes the texture of the tumor tenacious, which increases surgical difficulty $(12,13)$. No related complications occurred in any of the cases in the present study. Generally, embolization is suitable only for patients with definite tumor-feeding arteries, as shown by angiography, and only if it does not affect the normal blood supply (13). The tumor-feeding arteries are often located deeply on both sides of the tumor, while the large draining veins typically arise from the tumor surface (13). With careful observation, these vessels can be identified accurately during the operation and embolization can be performed with a high ratio of technical success, without permanent neurological complications.

Although 6-10\% of all intracranial tumors are found in the $\mathrm{CPA}$, the majority are vestibular schwannomas and meningiomas $(9,11)$. Because of differences in the surgical strategies, differential diagnosis is crucial for safe tumor management. Hemangioblastomas generally have an intra-axial origin and are rarely found in the CPA (13). To our knowledge, a very small number of patients with large hemangioblastoma in the CPA have been reported, and, of those, only 10 cases were presented in English literature $(2,7,9)$. One large tumor in the CPA was observed in one of the patients in the present study. The tumor was completely resected via a modified far-lateral approach. The embolized feeding arteries were visible in the microscopic fields during the operation as solid white vessels, thus becoming useful for intraoperative orientation. Postoperative MRI showed no residual tumor. Ataxia and nystagmus were not improved, but no newly developed cranial nerve deteriorations were observed. There were no operative complications.

Various approaches, including the suboccipital ipsilateral approach, the modified far-lateral approach and the suboccipital midline approach, have been used with or without preoperative embolization for the surgical resection of hemangioblastomas. About two-thirds of hemangioblastomas appear as well-circumscribed cystic masses with hypervascular mural nodules. Although there are no histological differences between the cystic and solid tumor subtypes, solid subtypes may require a more extended approach to achieve an adequate operation space $(6,11)$. Dow et al (11) used the transcochlear far lateral approach in patients with large $(>3 \mathrm{~cm})$ solid hemangioblastoma in the CPA. This approach provided visual access that was wide enough to permit early control of proximal feeding vessels and to allow a safe circumferential dissection of the lesion (11). For cystic hemangioblastomas in the CPA, Bush et al (9) removed the tumor via a translabyrinthine approach, as they first mistook it for an atypical cystic vestibular schwannoma. They suggested that, had an hemangioblastoma been considered, the suboccipital approach might have provided adequate exposure and potentially preserved the hearing of the patient (9). Therefore, novel microsurgical techniques and a better understanding of the vascular pattern of this type of tumor have enhanced the surgical strategies for hemangioblastoma.

In conclusion, given the improvements in microsurgical techniques and the understanding of the tumor vascular pattern, en bloc surgical resection is the optimal treatment for solid hemangioblastomas of the posterior fossa. For large tumors, selective preoperative embolization of the feeding artery is critical for tumor removal associated with a low morbidity and mortality rate.

\section{Acknowledgements}

This study was supported by the Science and Technology Commission Foundation of Shanghai (grant no. 11JC1408602), the Natural Science Foundation of China (grant nos. 81372710, 81000527, 81101801 and 81100547) and the Natural Science Foundation of Jiangsu Province (grant no. BK2010159). The authors would like to thank Clarity Manuscript Consultants LLC (Indianapolis, IN, USA) for technical assistance in editing this manuscript.

\section{References}

1. Amano T, Tokunaga S, Shono T, Mizoguchi M, Matsumoto K, Yoshida F and Sasaki T: Cerebellar haemangioblastoma manifesting as hearing disturbance. Neurol Med Chir (Tokyo) 49: 418-420, 2009. 
2. Rachinger J, Buslei R, Prell J, Strauss C and Strauss C: Solid haemangioblastomas of the CNS: A review of 17 consecutive cases. Neurosurg Rev 32: 37-47; discussion 47-48, 2009.

3. Roberti F, Jones RV and Wright DC: Cranial nerve haemangioblastomas. Report of a rare case and review of literature. Surg Neurol 67: 640-646; discussion 646, 2007.

4. Singounas EG: Haemangioblastomas of the central nervous system. Acta Neurochir (Wien) 44: 107-113, 1978

5. Kamitani H, Hirano N, Takigawa H, Yokota M, Miyata H, Ohama E and Watanabe T: Attenuation of vascularity by preoperative radiosurgery facilitates total removal of a hypervascular haemangioblastoma at the cerebello-pontine angle: Case report Surg Neurol 62: 238-243; discussion 243-244, 2004.

6. Matsushima T, Kawashima M, Masuoka J, Mineta T and Inoue T: Transcondylar fossa (supracondylar transjugular tubercle) approach: Anatomic basis for the approach, surgical procedures, and surgical experience. Skull Base 20: 83-91, 2010.

7. Nair BR, Joseph V, Chacko G and Keshava SN: Giant solid haemangioblastoma of the cerebellopontine angle: A technically challenging case. Neurol India 62: 228-229, 2014.
8. Wan JQ, Cui H and Wang Y: Surgical management of large solid haemangioblastomas of the posterior fossa. J Clin Neurosci 18 39-42, 2011.

9. Bush ML, Pritchett C, Packer M, Ray-Chaudhury A and Jacob A Haemangioblastoma of the cerebellopontine angle. Arch Otolaryngol Head Neck Surg 136: 734-738, 2010.

10. Biondi A, Ricciardi GK, Faillot T, Capelle L, Van Effenterre R and Chiras J: Haemangioblastomas of the lower spinal region: Report of four cases with preoperative embolization and review of the literature. AJNR Am J Neuroradiol 26: 936-945, 2005.

11. Dow GR, Sim DW and O'Sullivan MG: Excision of large solid haemangioblastomas of the cerebellopontine angle by a skull base approach. Br J Neurosurg 16: 168-171, 2002.

12. Cornelius JF, Saint-Maurice JP, Bresson D, George B and Houdart E: Hemorrhage after particle embolization of haemangioblastomas: Comparison of outcomes in spinal and cerebellar lesions. J Neurosurg 106: 994-998, 2007.

13. Montano N, Doglietto F, Pedicelli A, Albanese A, Lauretti L, Pallini R, Lauriola L, Fernandez E and Maira G: Embolization of haemangioblastomas. J Neurosurg 108: 1063-1064, 2008. 